\title{
Plasma density transition trapping as a possible high-brightness electron beam source
}

\author{
M. C. Thompson* and J. B. Rosenzweig \\ Department of Physics and Astronomy, University of California, Los Angeles, California 90095, USA
}

H. Suk

Korea Electrotechnology Research Institute, Changwon 641-120, Republic of Korea

(Received 11 February 2003; published 6 January 2004)

\begin{abstract}
Plasma density transition trapping is a recently proposed self-injection scheme for plasma wakefield accelerators. This technique uses a sharp downward plasma density transition to trap and accelerate background plasma electrons in a plasma wakefield. This paper examines the quality of electron beams captured using this scheme in terms of emittance, energy spread, and brightness. Two-dimensional particle-in-cell simulations show that these parameters can be optimized by manipulating the plasma density profile. We also develop, and support with simulations, a set of scaling laws that predicts how the brightness of transition trapping beams scales with the plasma density of the system. These scaling laws indicate that transition trapping can produce beams with brightness $\geq 5 \times 10^{14} \mathrm{~A} /(\mathrm{mrad})^{2}$. A proof-ofprinciple transition trapping experiment is planned for the near future. The proposed experiment is described in detail.
\end{abstract}

DOI: 10.1103/PhysRevSTAB.7.011301

PACS numbers: 52.40.Mj, 52.59.Sa

\section{INTRODUCTION}

In a plasma wakefield accelerator (PWFA) a short, high density electron beam is used to drive large amplitude plasma waves. Accelerating gradients in these systems scale with the nonrelativistic plasma frequency $\omega_{p}=$ $\left(4 \pi n_{0} e^{2} / m_{e}\right)^{1 / 2}$, where $n_{0}$ is the plasma density, $e$ is the electron charge, and $m_{e}$ is the electron mass. It follows that high gradient PWFAs have very short period waves. Accelerating a second beam in such a system and maintaining its energy spread and emittance requires injecting a subpicosecond beam into the drive beam's wake with well subpicosecond timing accuracy. This is often referred to as witness beam injection, which has never been fully achieved experimentally. All experiments to date that have injected external electrons into accelerating plasma waves have used either continuous electron beams or beam pulses that were long compared to the plasma wave [1-5]. As a result the accelerated electrons had induced energy spread equivalent to the acceleration, which would eventually result in 100\% energy spread.

The difficulty of witness beam injection makes it desirable to develop a system in which charge is automatically loaded into the accelerating portion of the wake by the drive beam's interaction with the static plasma environment. This approach allows timing concerns to be eliminated entirely. Bulanov et al. have suggested such a scheme for laser wakefield accelerators in which a region of gradually declining plasma density is used to produce plasma electron trapping through gentle conventional wave breaking [6]. Suk et al. [7] recently proposed a new self-trapping system for use in the blowout regime of PWFAs [8] where $n_{b}>n_{0}$ (underdense condition). In

*Electronic address: mct@physics.ucla.edu this scheme the beam passes though a sharp drop in plasma density where the length of the transition between the high density in region one (1) and the lower density in region two (2) is smaller than the plasma skin depth $k_{p}^{-1}=v_{b} / w_{p}$, where $v_{b} \cong c$ the driving pulse's velocity. As the drive beam's wake passes the sudden transition there is a period of time in which it spans both regions. The portion of the wake in region 2 has lower fields and a longer wavelength than the portion in region 1 . This means that a certain population of the plasma electrons at the boundary will suddenly find themselves rephased into an accelerating portion of the region 2 wake. When the parameters are correctly set, these rephased electrons are inserted far enough into the accelerating region to be trapped and subsequently accelerated to high energy.

The plasma density transition trapping scheme originally proposed by Suk et al., like the system presented by Bulanov et al., provides very short injection pulses that are phase locked to the plasma wave, but suffers from a lack of beam quality, as defined by energy spread and transverse emittance. We have found, however, that beam quality can be greatly enhanced in the plasma density transition trapping system by tailoring the density profile of the plasma and scaling to higher plasma density. When scaled to densities $\sim 10^{17} \mathrm{~cm}^{-3}$ the performance of transition trapping, as measured by beam brightness, surpasses state-of-the-art photoinjectors and is competitive with optically stimulated plasma injection systems $[9,10]$ operating at similar densities. The advantage of laser driven schemes is their inherently high density operation, which we will see below implies high-brightness beam production. Alternatively, transition trapping is a simpler scheme free of timing concerns, but has only been examined extensively at relatively low densities $\sim 10^{13} \mathrm{~cm}^{-3}$. If plasma density transition trapping can be successfully 
TABLE I. Drive and captured beam parameters in the strong blowout case. Figures for the captured beam are for the core of the captured beam, which is about $20 \%$ of the captured particles, after $12 \mathrm{~cm}$ of acceleration.

\begin{tabular}{cccc}
\hline \hline \multicolumn{2}{c}{ Drive beam } & \multicolumn{2}{c}{ Captured beam } \\
\hline Beam energy & $50 \mathrm{MeV}$ & Beam energy & $56 \mathrm{MeV}$ \\
Beam charge & $63 \mathrm{nC}$ & Beam charge & $5.9 \mathrm{nC}$ \\
Beam duration $\sigma_{t}$ & $3 \mathrm{ps}$ & Beam duration $\sigma_{t}$ & $161 \mathrm{fs}$ \\
Beam radius $\sigma_{r}$ & $500 \mu \mathrm{m}$ & Beam radius $\sigma_{r}$ & $112 \mu \mathrm{m}$ \\
Peak beam density & $1.2 \times 10^{14} \mathrm{~cm}^{-3}$ & Normalized emittance $\varepsilon_{x}$ & $155 \mathrm{~mm} \mathrm{mrad}$ \\
& & Total energy spread & $13 \%$ \\
\hline \hline
\end{tabular}

scaled to high densities it may provide a very simple and robust method of high-brightness beam generation.

In this paper, we expand on the original proposed transition trapping system, examining in greater detail the issues of trapped beam quality and the techniques for optimizing it. We also present a detailed plan for a plasma density transition trapping proof-of-principle experiment and report on substantial progress towards realizing this experiment.

\section{TRAPPING SCENARIOS}

The current development of the idea of plasma density transition trapping centers around the detailed study of two particular scenarios. The first case uses a high charge beam to create a very strong blowout of plasma electrons in a plasma with a simple step function longitudinal plasma density profile. This is the original case proposed for transition trapping [7]. The second case is optimized for a proof-of-principle trapping experiment. This case uses a beam of much more modest charge to create a weak blowout in the high density region, and uses a sloped plasma density profile to enhance charge capture and reduce energy spread.

\section{A. Strong blowout scenario}

The strong blowout scenario uses the parameters presented in Table I and illustrated in Fig. 1. The plasma density profile is a simple step function with a constant density of $n_{\text {region } 1}=5 \times 10^{13} \mathrm{~cm}^{-3}$ in the high density region and a constant density of $n_{\text {region } 2}=3.5 \times$ $10^{13} \mathrm{~cm}^{-3}$ in the low density region. The high charge driver produces a very strong blowout, which in turn results in a clear picture of the trapping process.

In order to increase our understanding of the trapping mechanism we performed a series of simulations with the 2D particle-in-cell code MAGIC [11] in which the high and low density plasma electron populations are tracked separately. The results show that the trapping process actually begins in the high density region, as can be seen in Fig. 1. As electrons from the low density region are blown out and pushed backward they enter the high density plasma region. There the oscillation of the region 2 plasma electrons is sped up by the higher ion density and these electrons return to the axis early to mix with electrons from the high density region. As this mixed concentration of plasma electrons crosses the boundary between the high and low density regions many of the electrons find themselves in an accelerating phase of the low density plasma wake and are trapped and accelerated.

The properties of the beam captured in this scenario are listed in the second column of Table I. The captured beam is very short and has a small radius, both of which originate from the small accelerating volume of the accelerating plasma wave. The beam also has a high charge that results from the very high concentration of electrons in the oscillation density spike that are injected. Unfortunately, the captured beam has a significant
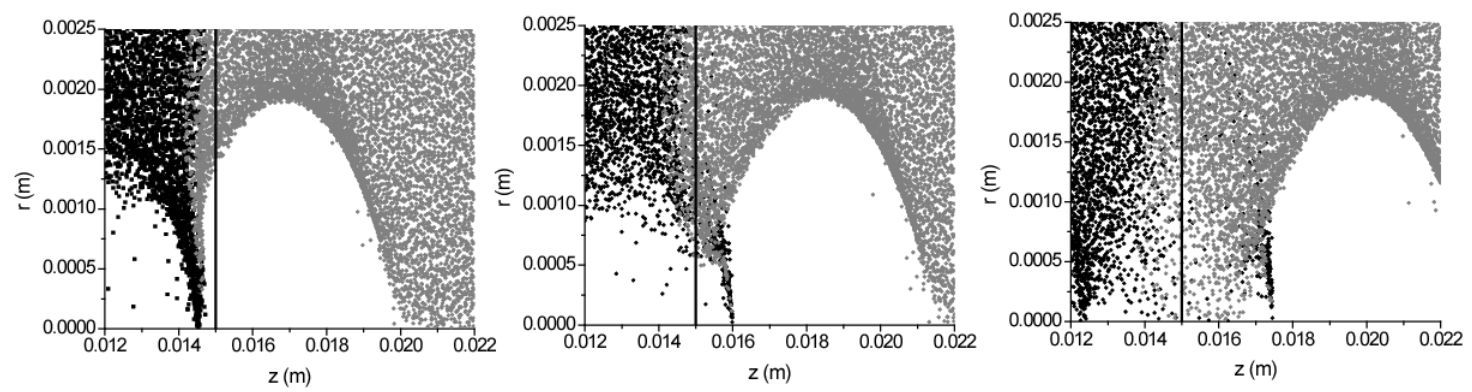

FIG. 1. Configuration space $(r, z)$ distributions of the plasma electrons illustrating trapping in the strong blowout case. The vertical black line indicates the original position of the density transition. Plasma electron particles originating in the high density region are colored black while particles originating in the low density region are colored gray. 
energy spread that results from the fast variation in the plasma wakefield accelerating gradient where the particles are captured. The beam also has a poor transverse emittance. This is an unavoidable consequence of trapping background plasma particles in the strong blowout regime. The large amplitudes of transverse momenta imparted to the plasma electrons as the drive beam space charge blows them out to the side remains with the particles as they are trapped and accelerated to high energy.

In addition to the undesirable emittance and energy spread properties of the captured beam, this transition trapping scenario is also impractical from an experimental standpoint. The drive beam parameters listed in the first column of Table I are not currently achievable. For this reason we began to look at what sort of trapping experiments could be done with the more modest driver beams that are available. During this development we also found ways to improve both the emittance and energy spread of the captured beams.

\section{B. Weak blowout scenario}

A great deal can be learned about the mechanism and dynamics of density transition trapping by comparing the strong blowout case previously described to a case in which a weak blowout is used, as illustrated in Fig. 2 . Our standard example of a weak blowout case is the proof-of-principle experimental case designed for the
Neptune Advanced Accelerator Laboratory at UCLA [12]. This case was developed and optimized for parameters achievable at the Neptune Laboratory through extensive simulations with MAGIC. The driving beam parameters of the simulation are shown in Table II. The driving beam has a ramped longitudinal profile as shown in Fig. 3. Ramped profiles of this type maximize the transformer ratio of the wakefield [13] and can be produced using a negative $R_{56}$ magnet compressor system. We are developing such a compressor system for the Neptune Laboratory [14]. While the ramped beam profile improves performance, it is not critical to this trapping scenario.

The plasma density profile used in this case is illustrated in Fig. 3. The plasma density profile is tailored to maximize the amount of charge captured while maintaining an acceptable amount of acceleration. The first $\mathrm{cm}$ of the profile reflects a realistic finite rise time from zero to the maximum plasma density. After $5 \mathrm{~mm}$ of maximum density the transition takes place and the density is reduced to $18 \%$ of the maximum. This density drop is near the optimum to maximize charge capture. Decreasing the density of region 2 increases the wavelength of the accelerating plasma wave. This has the effect of enlarging the volume of the capture region and enhancing the amount of charge trapped. Lowering the plasma density also reduces the accelerating gradient, however, reducing the number of initially captured particles that ultimately achieve resonance with the accelerating wave. These two effects compete with the charge capture maximum

TABLE II. Drive and captured beam parameters in the weak blowout case.

\begin{tabular}{cccc}
\hline \hline \multicolumn{1}{c}{ Drive beam } & \multicolumn{2}{c}{ Captured beam } \\
\hline Beam energy & $14 \mathrm{MeV}$ & Beam energy & $1.2 \mathrm{MeV}$ \\
Beam charge & $5.9 \mathrm{nC}$ & Beam charge & $120 \mathrm{pC}$ \\
Beam duration & $6 \mathrm{ps}$ & Beam duration $\sigma_{t}$ & $1 \mathrm{ps}$ \\
Beam radius $\sigma_{r}$ & $540 \mu \mathrm{m}$ & Beam radius $\sigma_{r}$ & $380 \mu \mathrm{m}$ \\
Normalized emittance $\varepsilon_{x}$ & $15 \mathrm{~mm} \mathrm{mrad}$ & Normalized emittance $\varepsilon_{x}$ & $15 \mathrm{~mm} \mathrm{mrad}$ \\
Peak beam density & $4 \times 10^{13} \mathrm{~cm}^{-3}$ & Total energy spread & $11 \%$ \\
\hline \hline
\end{tabular}
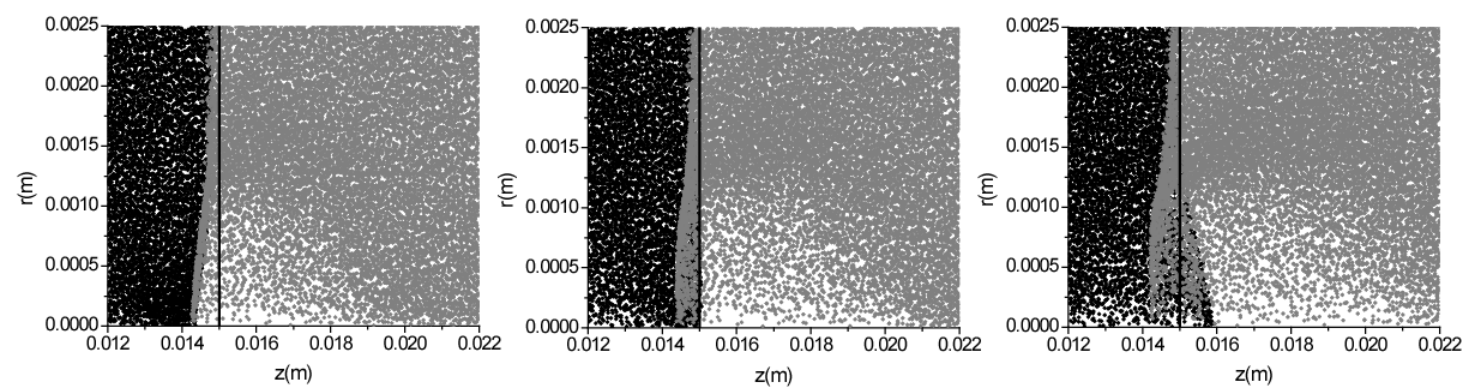

FIG. 2. Configuration space $(r, z)$ distributions of the plasma electrons illustrating trapping in the weak blowout case. This figure is directly comparable to Fig. 1. The scale and particle coloring are identical. Note that the trapping mechanism is essentially the same except that it proceeds more slowly due to the low plasma density in the downstream region compared to the strong blowout case. The weaker blowout also leads to much less transverse disturbance in the plasma, which in turn yields lower emittance. 

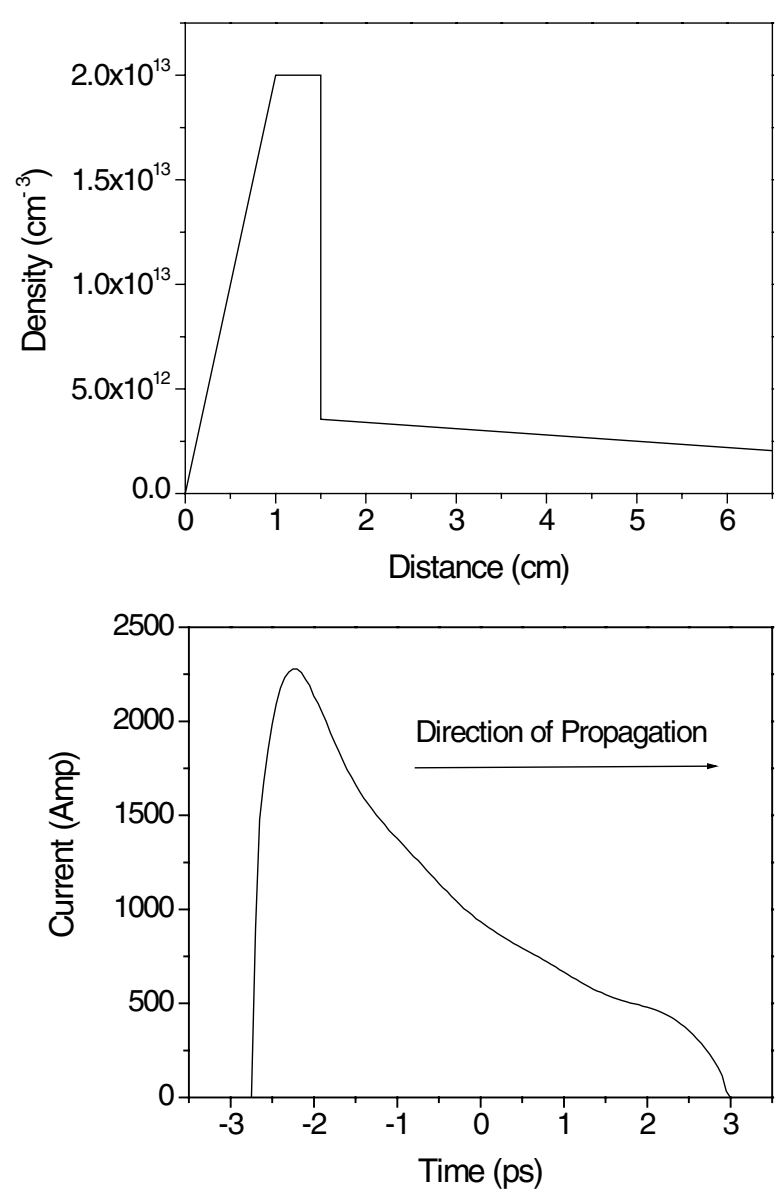

FIG. 3. Plasma density (top) and drive beam current (bottom) profiles.

occurring at $n_{\text {region 2 }}=0.18 n_{\text {region } 1}$. To quantify the degree of blowout in this case, we note that the electron beam density is 2 times larger than the peak plasma density of $2 \times 10^{13} \mathrm{~cm}^{-3}$, as can been seen from Table II and Fig. 3.

In the simulation the transition is approximated as a perfect step function; the validity of this assumption will be elaborated on later in this paper. Finally, the gradual decline in plasma density after the transition slowly increases the plasma wavelength, and thus the extent of the accelerating phase of the wakefield region. The growth in plasma wavelength reduces the peak gradient but rephases the captured charge forward of the peak field of the wake into a region of slightly weaker, but more uniform, acceleration. This rephasing both increases the amount of charge trapped and reduces energy spread. The rate of density decline can be increased to reduce energy spread even further. Gradually declining post transition plasma densities have been shown to have similar benefits in the strong blowout regime [15].

The parameters of the bunch of captured plasma electrons are given in Table II. The captured plasma electrons form a well-defined beam of substantial charge that can be propagated and detected without major difficulty. The captured beam is also well separated from the drive beam in energy and should be easy to isolate. If a bi-Gaussian beam with $\sigma_{t}=1.5 \mathrm{ps}$ is substituted for the ramped profile shown in Fig. 3, the captured beam parameters remain unchanged except for a $20 \%$ loss of captured charge.

\section{EXPERIMENTAL PLANS}

An experiment is planned with the parameters presented in Table II and Fig. 3. Originally planned for the Neptune Laboratory at UCLA, this experiment will be performed at the Fermilab NICADD Photoinjector Laboratory (FNPL) as part of our larger UCLA/FNAL collaboration on PWFAs. The FNPL accelerator is an $18 \mathrm{MeV}$ electron linac [16]. The system consists of a normal conducting $L$-band $\mathrm{rf}$ gun with a cesium telluride photocathode and a 9-cell superconducting accelerating cavity. Bunches with charge in excess of $8 \mathrm{nC}$ can be produced and compressed to durations of $1.6 \mathrm{ps}$ rms using magnetic compression. By modifying an existing pulse discharge plasma source [17] we have created a plasma column with a peak density of $6 \times 10^{13} \mathrm{~cm}^{-3}$. As shown in Fig. 4, the raw plasma column has a Gaussian transverse density profile and over $6 \mathrm{~cm}$ of the plasma has density greater than $2 \times 10^{13} \mathrm{~cm}^{-3}$, the required peak density for the trapping experiment.

Experimental realization of plasma density transition trapping depends on the creation of sharp density transitions. The limit on the sharpness of the transition necessary to produce trapping is set by the trapping condition

$$
k_{p}^{\text {region } 1} L_{\text {trans }}<1
$$

As can be seen from Fig. 5, this is a very strict condition. The turn on of the capture in this regime is nearly a step function.

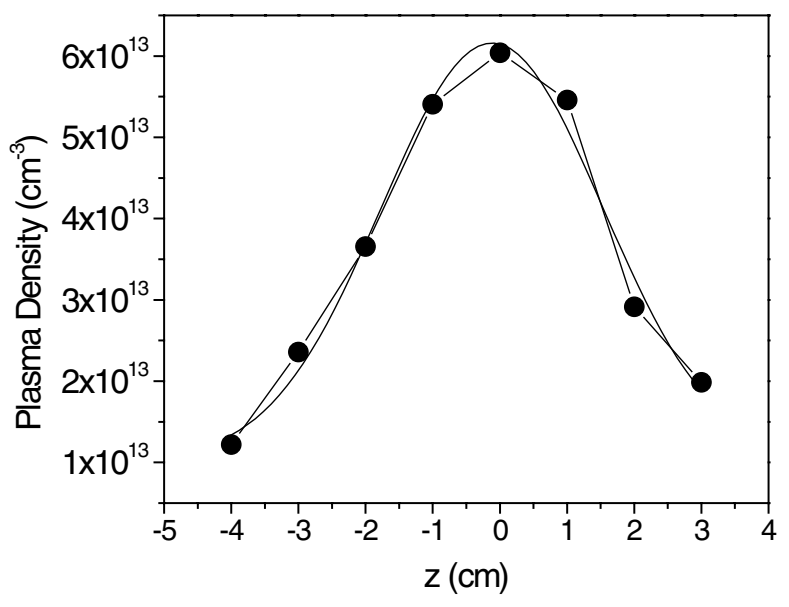

FIG. 4. Measured transverse density profile of the plasma column. 


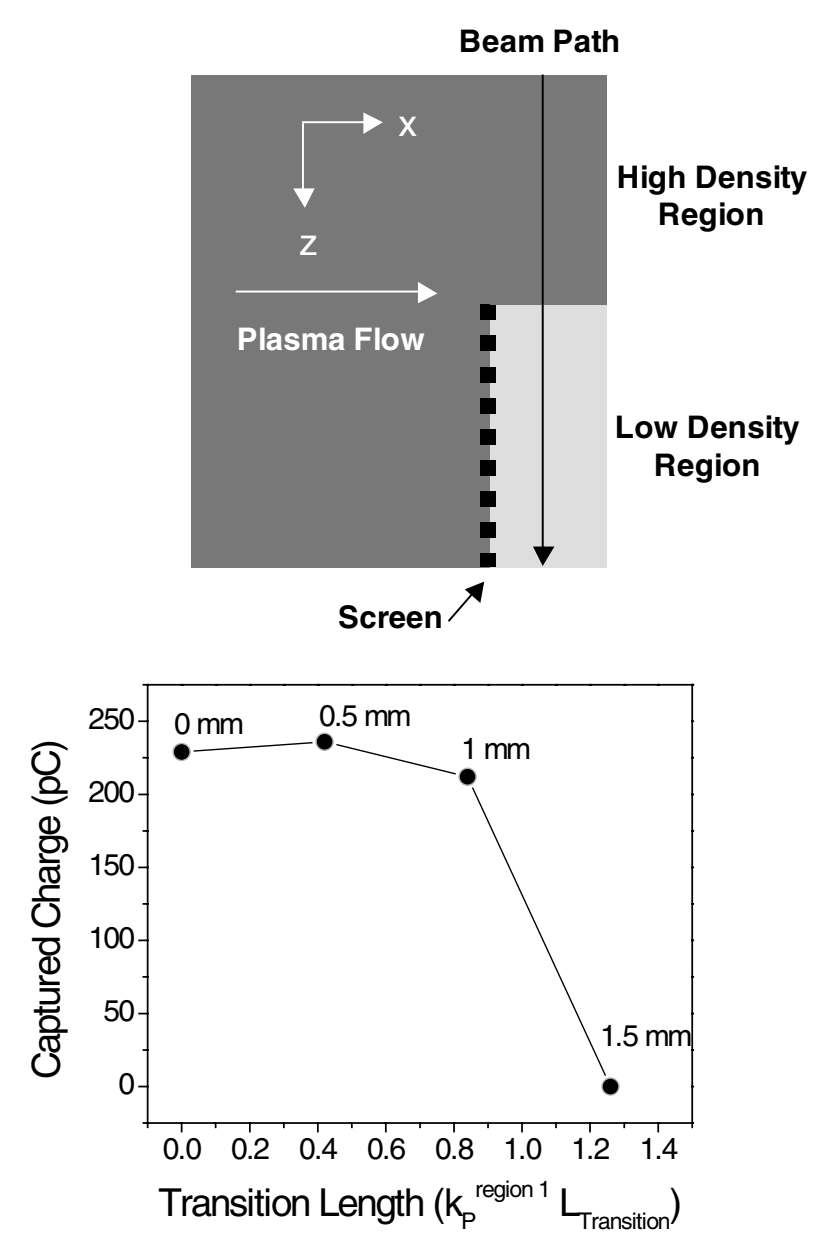

FIG. 5. Top: Simplified diagram of a plasma density transition produced by a obstructing screen. Bottom: Simulated dependence of captured charge on transition length in the proposed experimental case. Each point is marked with the length of the transition.

The creation of a density transition that satisfies this criteria is an interesting experimental challenge. At high plasma densities, $n \geq 10^{14} \mathrm{~cm}^{-3}$, it will probably be necessary to directly create the plasma with the required density profile already built in. This might be accomplished though photoionization using a laser with an intensity profile that matches the desired plasma density profile or by using a uniform laser to ionize a dual density gas jet. At lower densities such as $10^{13} \mathrm{~cm}^{-3}$, which can be easily produced using discharges, it is possible to consider a simpler method using a masking screen to generate the density transition. The basic concept of operation for the masking screen is illustrated in the top portion of Fig. 5. Consider a system in which the plasma discharge is separated from the path of the driver beam. Once the plasma is created in the discharge apparatus it will diffuse and flow towards the beam path. If a perforated metal foil or grid of wires is placed in the path of the plasma flow it will block a portion of the flow creating a low density region. Unfortunately, the plasma density transition will not remain sharp as the distance from the screen grows as portrayed in the simple picture of Fig. 5. In reality the two plasma regions will diffuse into one another on the far side of the screen so that the plasma density transition will lengthen and blur as the distance from the screen edge increases. This process can be quantified using a simple model based on the velocities with which the plasma diffuses, as shown in Fig. 6. On the far side of the screen from the plasma source the high density plasma will continue to flow past the screen in the direction of the bulk plasma flow with a velocity $V_{\|}$and will begin flowing into the low density region with a velocity $V_{\perp}$. The sum of these two vectors defines the line which marks the end of the transition into the low density plasma region. Symmetry dictates that the start of the transition in the high density region can be defined in the same way so that the total transition length is given by

$$
L_{\text {trans }}=2 x \tan \theta=2 x \frac{V_{\perp}}{V_{\|}} .
$$

Since our plasma is weakly magnetized it is reasonable to assume that the parallel and perpendicular plasma flow velocities are approximately equal. This assumption leads to the conclusion

$$
V_{\perp} \approx V_{\|} \rightarrow L_{\text {trans }}=2 x,
$$

which in turn leads to a new experimental constraint on achieving efficient trapping,

$$
x<\frac{k_{p}^{-1}}{2} .
$$

This new trapping condition for obstructing screens requires that the drive beam passes within half a plasma skin depth of the boundary. For a $2 \times 10^{13} \mathrm{~cm}^{-3}$ plasma the drive beam will have to pass within $600 \mu \mathrm{m}$ of the screen. This level of pointing accuracy and stability is not difficult to achieve.

We have explored the validity of this model through simulations and experiment. The results of MAGIC PIC simulations of the screen-plasma interaction match the predictions of Eq. (3) almost exactly. Early experiments with metal screens in the plasma verified the density

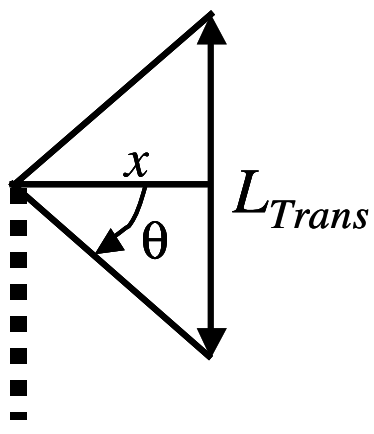

\section{Plasma Flow Velocities}

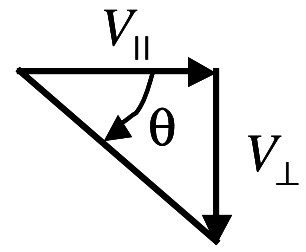

FIG. 6. Illustration of the transition geometry. 


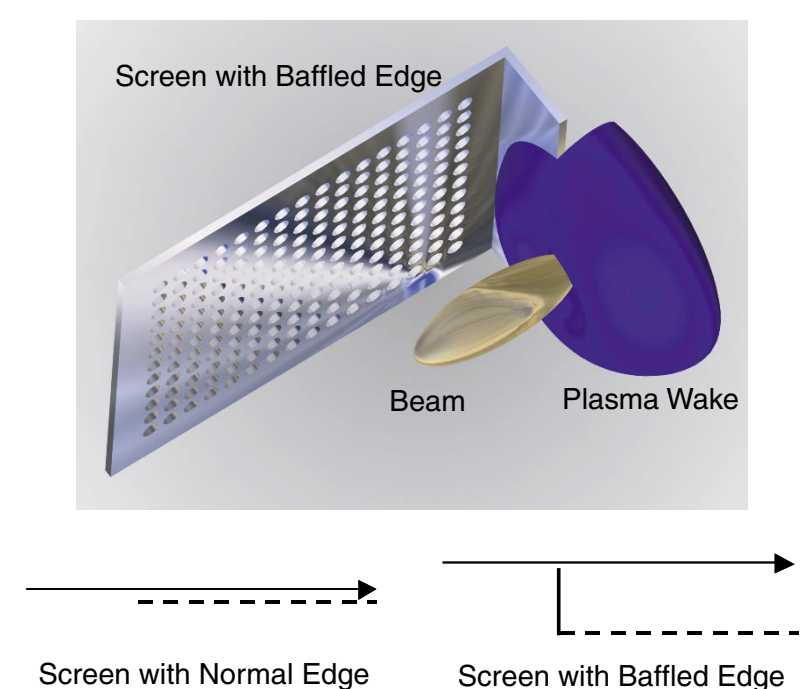

FIG. 7. (Color) Artist's concept of partial blocking of wake particles by the baffle. The prolate spheroid represents the electron beam, and the toroid that partially intersects the screen baffle is the plasma wake.

masking effect, but measurements of the density transition were inconclusive due to inadequate positioning equipment. A set of high precision density transition measurements is planned.

Propagating a beam so close to a metallic screen leads to other difficulties. Interactions with the screen over the entire length of the low density plasma region will completely disrupt the processes of trapping and acceleration. To circumvent this problem we examined many alternative geometries and arrived at a solution based on a screen with a solid metal baffle attached to its edge. As shown in Fig. 7 this baffle moves the sharp portion of the density transition away from the screen so that the beam and plasma wake will no longer interact with it. During the trapping process at the transition, however, the beam and wake still interact with the baffle. The primary effect of the baffle is to block a portion of the particles participating in the plasma wake oscillation, as illustrated on the top in Fig. 7.

Simulating the effects of the baffle on particle trapping is a complex problem. The baffle breaks the cylindrical symmetry of the problem requiring that any simulations of its effects must be done in three dimensions. We used the three-dimensional version of the PIC code MAGIC to simulate this problem. We modeled the trapping system with a metallic baffle at various distances from the beam center. The results of these simulations are summarized in Fig. 8. The points in the graph are taken from simulations in which the simulation cells are $0.17 k_{p}^{-1}$ on a side, which is the maximum resolution we could obtain with the code and our computing hardware.

Since Eq. (4) indicates that the beam must pass within $k_{p}^{-1} / 2$ of the baffle edge, the results shown in Fig. 8

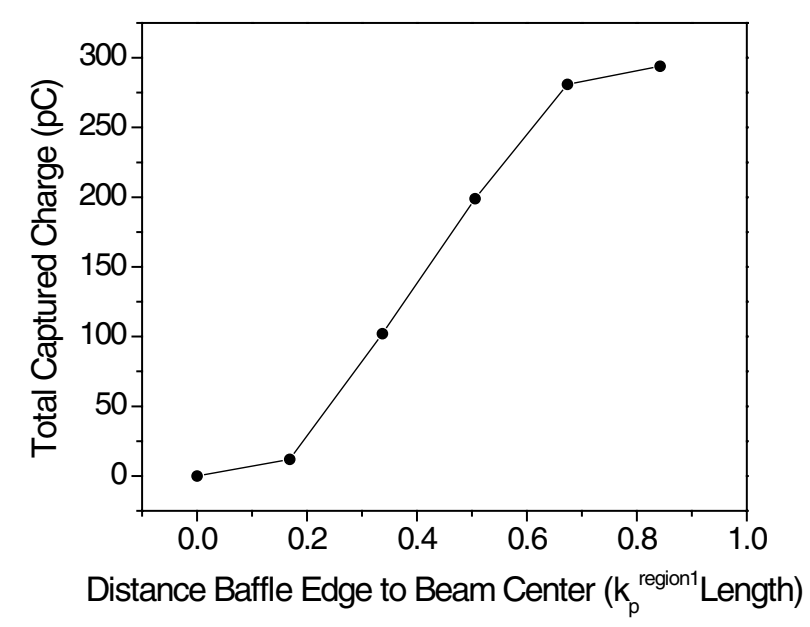

FIG. 8. Effect of the beam-baffle distance on trapping.

predict an approximate 50\% loss of total captured charge. This may not translate into a 50\% loss of particles in the beam core, however, since the large amplitude particles blocked by the baffle are not necessarily the ones that form the beam core. The 3D simulations lacked the resolution to resolve this question.

The final issue with the use of screen produced plasma density transitions is the rapid growth of the transition length with distance from the screen. The growth rate is large enough that there will be a significant transition length gradient over the distance spanned by the plasma wakefield. The effect of this transition length gradient is unknown, but will soon be examined in simulation. We expect this effect to produce another minor, but acceptable, degradation of the trapping performance.

\section{DRIVER CHARGE SCALING}

While the captured beam parameters presented in Table II are adequate for a first step, proof-of-principletype experiment, they are far inferior to the state-of-theart beams produced in modern photoinjectors. In order to find a systematic way to improve the captured beam parameters we began to simulate the effects of scaling up the drive beam charge without altering the rest of the experiment. The results of these simulation are shown in Fig. 9. Increasing the driver charge increases the strength of the blowout forming a larger amplitude more nonlinear plasma wave. It follows that all the accelerating fields in the problem are increased, as is the size of the accelerating wave. The impact on the captured beam is clearly shown in Fig. 9. The amount of charge captured, the length of the beam, and the emittance all grow as the driver charge is increased. Although it deserves more detailed study, simple scaling of the driver charge appears to lead to bigger captured beams but not higher quality ones. 

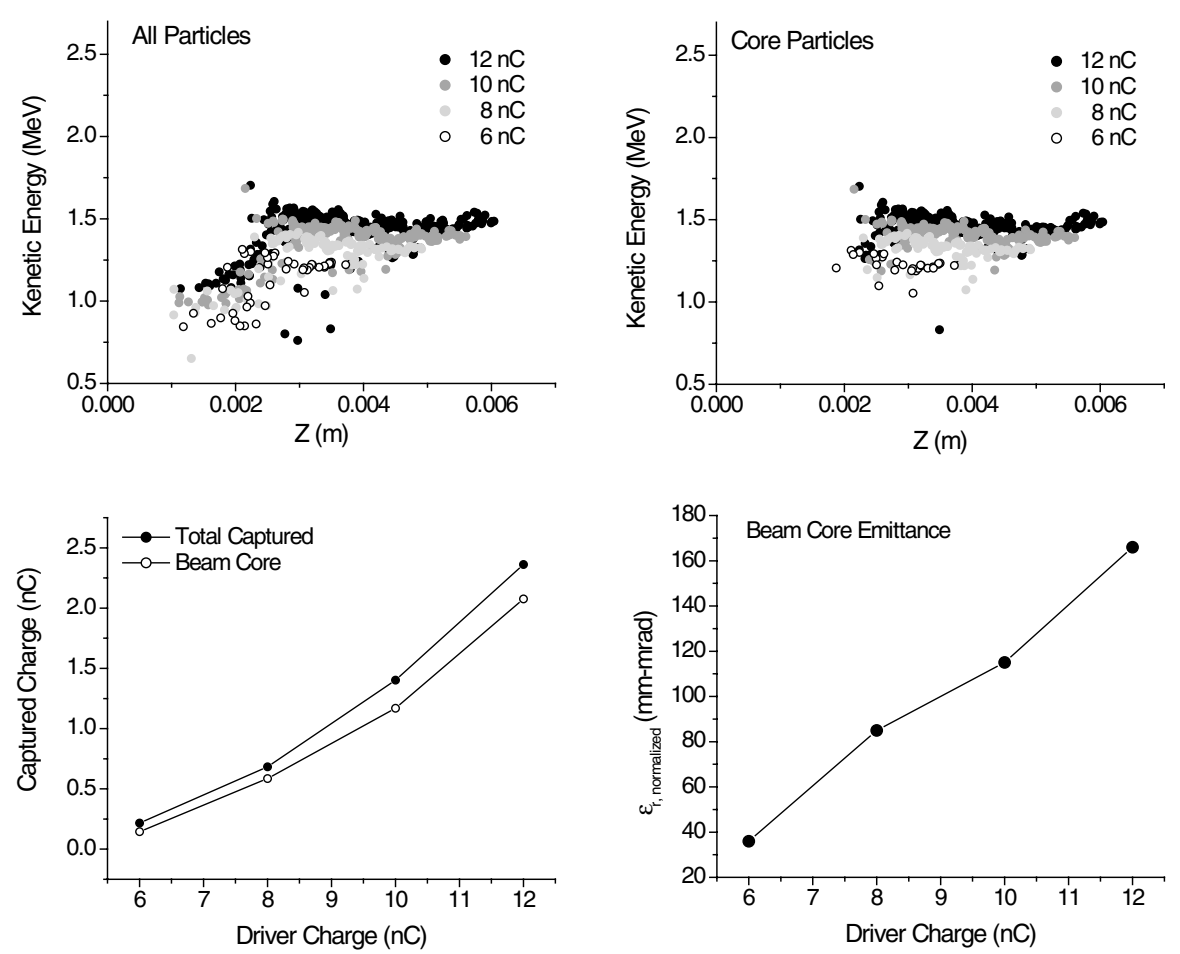

FIG. 9. Variation of the captured beam parameters with scaling of the driver beam charge in the weak blowout case.

\section{WAVELENGTH SCALED SOURCES}

We have seen the performance of density transition trapping at densities $n_{0} \sim 10^{13} \mathrm{~cm}^{-3}$ in the preceding sections, as well as how the performance changes with driver charge scaling. From these studies it is clear that transition trapping at $n_{0} \sim 10^{13} \mathrm{~cm}^{-3}$ produces beams of low brightness when compared to the benchmark of the Linac Coherent Light Source (LCLS) photoinjector [18]; see Table III. It is therefore interesting to examine how the captured beam performance scales with plasma density or, equivalently, the plasma wavelength. This type of wavelength scaling, and its impact on beam emittance and brightness, has been previously examined in the context of rf acceleration [19] in photoinjector sources, where the beam displays palsma-type behavior.

In order to scale the transition trapping system to a higher plasma density $n_{\text {high }}$ all the charge densities in the system must be increased by the ratio

$$
n_{\text {high }} / n_{0} \text {, }
$$

and all the lengths in the system are decreased by the ratio

$$
\frac{\lambda_{p \text { high }}}{\lambda_{p 0}}=\frac{k_{p \text { high }}^{-1}}{k_{p 0}^{-1}}=\frac{1 / \sqrt{n_{\text {high }}}}{1 / \sqrt{n_{0}}}=\sqrt{\frac{n_{0}}{n_{\text {high }}}},
$$

where $\lambda_{p}$ represents the typical wavelength of oscillations in the plasma and is equal to the plasma skin depth, $\lambda_{p}=$ $k_{p}^{-1}=c / \omega_{p}$. In scaling the system we also require that the plasma disturbance be self-similar. This means that both the relative density disturbance $\delta n / n_{0}$ and the normalized peak field $E_{z} / E_{\text {wave break }}=e E_{z} / m_{e} \omega_{p} c$ remain constant. It follows that the scaled phase space distributions of the captured electrons will also be self-similar. This can be seen from noting that the above requirement can be written as

$$
\frac{e E_{z}}{m_{e} \omega_{p} c}=\frac{e}{m_{e} c^{2}} E_{z} \lambda_{p}=\text { const }
$$

\begin{tabular}{|c|c|c|c|c|c|c|c|}
\hline Peak density & $\sigma_{t, \text { driver }}$ & $Q_{\text {driver }}$ & $\sigma_{t, \text { trap }}$ & $Q_{\text {trap }}$ & $I_{\text {peak,trap }}$ & $\varepsilon_{x, \text { norm,trap }}$ & $B_{\text {norm, trap }}$ \\
\hline $2 \times 10^{13} \mathrm{~cm}^{-3}$ & $1.5 \mathrm{ps}$ & $10 \mathrm{nC}$ & $2.7 \mathrm{ps}$ & $1.2 \mathrm{nC}$ & $163 \mathrm{~A}$ & $57 \mathrm{~mm} \mathrm{mrad}$ & $5 \times 10^{10}$ \\
\hline $2 \times 10^{15} \mathrm{~cm}^{-3}$ & $150 \mathrm{fs}$ & $1 \mathrm{nC}$ & $270 \mathrm{fs}$ & $120 \mathrm{pC}$ & $166 \mathrm{~A}$ & $5.9 \mathrm{~mm} \mathrm{mrad}$ & $5 \times 10^{12}$ \\
\hline $2 \times 10^{17} \mathrm{~cm}^{-3}$ & $15 \mathrm{fs}$ & $100 \mathrm{pC}$ & $28 \mathrm{fs}$ & $12 \mathrm{pC}$ & $166 \mathrm{~A}$ & $0.6 \mathrm{~mm} \mathrm{mrad}$ & $5 \times 10^{14}$ \\
\hline \multicolumn{5}{|c|}{ LCLS photoinjector specification } & $100 \mathrm{~A}$ & $0.6 \mathrm{~mm} \mathrm{mrad}$ & $2.8 \times 10^{14}$ \\
\hline
\end{tabular}

so that the captured particle momenta $p$ is given by

TABLE III. Simulations of wavelength scaling using MAGIC 2D. 


$$
p \propto E_{z} \lambda_{p}=\text { const. }
$$

Consequently, the emittance $\varepsilon$, which is proportional to the product of momenta and the beam size, goes like

$$
\varepsilon \propto \lambda_{p} p \propto \lambda_{p} .
$$

The emittance of the captured beam improves as the system is scaled to higher density as a result of the reduction in the transverse beam size.

The amount of charge captured $Q$ depends on both the available plasma electron density $n_{0}$ and the volume of the accelerating portion of the wave, which is proportional to $\lambda_{p}^{3}$. This scaling can be written as

$$
Q \propto n_{0} \lambda_{p}^{3} \propto n_{0}\left(\frac{1}{\sqrt{n_{0}}}\right)^{2} \lambda_{p} \propto \lambda_{p} .
$$

While the captured charge goes down as the plasma wavelength is reduced, the current $I$ remains constant since the length of the beam also goes down with the plasma wavelength,

$$
I \propto \frac{Q}{\lambda_{p} / c}=\text { const. }
$$

Finally, we can combine the scaling laws for emittance and current to deduce the scaling of the beam brightness $B$,

$$
B \propto \frac{I}{\varepsilon^{2}} \propto \frac{1}{\lambda_{p}^{2}} \propto n_{0} .
$$

Thus the brightness of electron beams produced using density transition trapping increases linearly with the density of the plasma.

These scaling laws were tested using the 2D PIC code MAGIC. The cases examined are scaled versions of the proof-of-principle experimental case with a slightly larger driver charge. The results are summarized in Table III. The simulation results follow the scaling laws precisely in the range studied. At $2 \times 10^{17} \mathrm{~cm}^{-3}$ transition trapping can produce an extremely short beam with excellent emittance and a brightness that exceeds stateof-the-art photoinjectors. The drive beams needed at all densities must be of similar length and approximately 1 order of magnitude greater charge than the beams they capture. The emittance of the driver, however, is irrelevant as long as the driving beam can be focused sufficiently to match into the plasma. This means that plasma density transition trapping might be used as an emittance transformer to produce short, low emittance beams from short beams with high emittances that were produced using extreme magnetic compression or other techniques that produce significant emittance growth. The feasibility of this idea is still under study and may be enhanced by our effort to find new scenarios that produce low emittance trapped beams.
As described previously, plasma density transition trapping, at least in the regimes examined so far, produces beams of large emittance due to the sizable transverse momenta the plasma particles have at capture. Scaling to higher density improves the emittance by reducing the beam size rather than reducing the transverse momentum. We are continuing to explore alternative transition trapping scenarios in an effort to reduce the transverse momentum of the beam further. This may be accomplished by using drive beams that are wide and or long compared to the plasma skin depth. The development of the technique of foil trapping, which is discussed in the next section, might also lead to lower transverse momenta.

\section{FUTURE DIRECTIONS}

In order to proceed beyond a proof-of-principle transition trapping experiment will necessarily require scaling to higher plasma densities. This will require improvements to both the driver beam and higher density plasma sources with sharp transitions. The production of very short, high current electron drive beams is a matter discussed at great length elsewhere. High power laser pulses are also being considered as alternative drivers for transition trapping [15]. Ideas for producing plasmas with transition that satisfy Eq. (1) at high densities $n \geq$ $10^{14} \mathrm{~cm}^{-3}$ are still in the conceptual phase. Possible techniques for producing these transitions include laser ionization of a dual density gas jet and photoionization of lithium using a laser with a step function intensity profile.

In the extreme limit, one can imagine creating an ultrasharp transition into a plasma by simply replacing the high density plasma region in a transition trapping scenario with a solid metal foil. Electrons would be provided for trapping from the foil via FowlerNordheim field emission [20]. Since this situation is much easier to produce experimentally than sharp plasma density drops, we have begun to look at the idea closely.

The field values necessary for significant FowlerNordheim emission are easy to achieve in current plasma wakefield experiments. Barov et al. have produced wakefields $\geq 140 \mathrm{MeV} / \mathrm{m}$ in a $10^{14} \mathrm{~cm}^{-3}$ plasma at FNAL [21]. In this experiment the drive beam enters the plasma through a metal foil, one side of which is immersed in the plasma and experiences the large plasma fields. Taking a reasonable value of $\beta \geq 50$ for the microscope surface field enhancement factor of the foil, Fowler-Nordheim theory predicts a large emission $J \geq 100 \mathrm{~A} / \mathrm{mm}^{2}$ under these conditions. Unfortunately, the emission of charge does not guarantee that the emitted charge will be trapped and accelerated. The charges emitted from the foil due to the plasma wakefields start essentially at rest and must be accelerated to resonance with the wave within the same period of the plasma wake. This situation is analogous to that in $\mathrm{rf}$ photoinjectors and the same dimensionless parameter can be used to evaluate the plasma wake's 
TABLE IV. Comparison of $\alpha$ parameters.

\begin{tabular}{lcccc}
\hline \hline \multicolumn{1}{c}{ Accelerating structure } & $E_{\max }$ & Frequency & $v_{\phi}$ & $\alpha$ \\
\hline 1.6 cell photoinjector & $80 \mathrm{MeV} / \mathrm{m}$ & $2.856 \mathrm{Ghz}$ & $c$ & 2.6 \\
Barov et al. wakefield experiment $(7 \mathrm{nC})$ & $300 \mathrm{MeV} / \mathrm{m}$ & $90 \mathrm{Ghz}$ & $c$ & 0.3 \\
Experiment with high charge driver $(70 \mathrm{nC})$ & $1.5 \mathrm{GeV} / \mathrm{m}$ & $90 \mathrm{Ghz}$ & $c$ & 1.6 \\
\hline \hline
\end{tabular}

potential to capture foil electrons. This parameter $\alpha$ is the ratio of the maximum normalized energy gain per unit length and the wave number of the accelerating wave

$$
\alpha=\frac{q E_{\max }}{k_{z} m_{e} c^{2}}=\frac{\left.\frac{d \gamma}{d z}\right|_{\max }}{k_{z}},
$$

where $k_{z}=\omega / v_{\phi}$. The capture of electrons starting from rest typically requires $\alpha \geq 1$. If we compare the $\alpha$ parameters of the Barov et al. experiment and a standard 1.6 cell photoinjector, see Table IV, we see that a plasma wake is not capable of capturing charge from a foil in this regime since its $\alpha$ is only 0.3 . The frequency of the accelerating wave is too high in comparison to the accelerating field and the emitted particles cannot achieve resonance with the wave.

The peak accelerating field can be increased by increasing the driver beam charge. If this is done while holding the plasma density constant, the plasma frequency will remain essentially unchanged and $\alpha$ will increase. The driver charge can be increased to the point where $\alpha>1$ and charge is captured from the foil in the plasma wake. If the driver charge in the Barov et al. experiment is increased by a factor of 10 the $\alpha$ of the system reaches 1.6 and charge is captured. The trapping behavior predicted by the $\alpha$ parameter has been verified by initial MAGIC 2D simulations. Further work needs to be done to explore the parameter space of foil trapping and characterize the captured beams.

\section{CONCLUSIONS}

The theoretical understanding of the basic plasma density transition trapping mechanisms are well developed. The quality of the beams produced by transition trapping is also well understood, as are a number of methods for optimizing the beam quality. We have shown, through the development of the wavelength scaling laws, that at high densities transition trapping can produce beams with brightness $\geq 5 \times 10^{14} \mathrm{~A} /(\mathrm{m} \mathrm{rad})^{2}$. This study shows an explicit, quantitative pathway toward ultrahigh brightness, small energy spread beam production using electron beam driven wakefield trapping which is competitive with state-of-the-art photoinjectors. Many variations on the idea of transition trapping, such as foil trapping and the use of drive beams that are long and or wide compared to the plasma skin depth, remain to be studied. In addition, many of the ideas developed in this paper, such as scaling to higher density and the use of gradually declin- ing plasma density profiles, may be applicable to other classes of plasma-based electron beam injectors $[9,10]$.

A detailed plan is in place for a proof-of-principle plasma density transition trapping experiment. This experiment will be conducted at low density using density modifying screens, a technique which we have made substantial progress in developing.

With further research and refinement plasma density transition trapping holds promise as a future highbrightness beam source. This source may take several forms such as an automatically timed PWFA injector or an "emittance transformer" used to convert short beams with poor emittance into short beams with a much lower transverse emittance.

\section{ACKNOWLEDGMENTS}

The authors thank T. C. Katsouleas and L. Ludeking for their assistance with the MAGIC PIC simulations. The authors also thank Mike Schneider, Chris Muller, Soren Telfer, and Ronald Agustsson for their technical assistance. This work was supported by the U.S. Department of Energy Grant No. DE-FG03-92ER40693.

[1] J. B. Rosenzweig, D. B. Cline, B. Cole, H. Figueroa, W. Gai, R. Konecny, J. Norem, P. Schoessow, and J. Simpson, Phys. Rev. Lett. 61, 98 (1988).

[2] C. E. Clayton, K. A. Marsh, A. Dyson, M. Everett, A. Lal, W. P. Leemans, R. Williams, and C. Joshi, Phys. Rev. Lett. 70, 37 (1993).

[3] F. Amiranoff et al., Phys. Rev. Lett. 81, 995 (1998).

[4] N. Barov, J. Rosenzweig, M. Conde, W. Gai, and J. G. Power, Phys. Rev. ST Accel. Beams 3, 011301 (2000).

[5] M. Hogan et al., Phys. Plasmas 7, 2241 (2000).

[6] S. Bulanov, N. Naumova, F. Pegoraro, and J. Sakai, Phys. Rev. E 58, R5257 (1998).

[7] H. Suk, N. Barov, J. B. Rosenzweig, and E. Esarey, Phys. Rev. Lett. 86, 1011 (2001).

[8] J. Rosenzweig, B. Breizman, T. Katsouleas, and J. Su, Phys. Rev. A 44, R6189 (1991).

[9] D. Umstadter, J. Kim, and E. Dodd, Phys. Rev. Lett. 76, 2073 (1996).

[10] E. Esarey, R. Hubbard, W. Leemans, A. Ting, and P. Sprangle, Phys. Rev. Lett. 79, 2682 (1997).

[11] B. Goplen, L. Ludeking, D. Smithe, and G. Warren, Comput. Phys. Commun. 87, 54 (1995).

[12] M. C. Thompson, C. E. Clayton, J. England, J. B. Rosenzweig, and H. Suk, in Proceedings of the 2001 
Particle Accelerator Conference, Chicago, Illinois (IEEE, Piscataway, NJ, 2001), p. 4014.

[13] P. Chen, J. J. Su, J. M. Dawson, K. L. Bane, and P. B. Wilson, Phys. Rev. Lett. 56, 1252 (1986).

[14] R. J. England, J. B. Rosenzweig, and M. C. Thompson, in Advanced Accelerator Concepts: Tenth Workshop, edited by Christopher E. Clayton and Patrick Muggli, AIP Conf. Proc. No. 647 (AIP, New York, 2002), p. 884.

[15] H. Suk, J. Appl. Phys. 91, 487 (2002).

[16] J.-P. Carneiro et al., in Proceedings of the 1999 Particle Accelerator Conference, New York, New York (IEEE, Piscataway, NJ, 1999), p. 2027.

[17] H. Suk, C. E. Clayton, G. Hairapetian, C. Joshi, M. Loh, P. Muggli, R. Narang, C. Pellegrini, J. B. Rosenzweig, and T. C. Katsouleas, in Proceedings of the 1999 Particle
Accelerator Conference, New York, New York (Ref. [16]), p. 3708 .

[18] M. Ferrario et al., in Proceedings of the European Particle Accelerator Conference 2000, Vienna, Austria (CERN, Geneva, 2000), p. 1642.

[19] J. Rosenzweig and E. Colby, in Advanced Accelerator Concepts, edited by Paul Schoessow, AIP Conf. Proc. No. 335 (AIP, New York, 1995), p. 724.

[20] R. H. Fowler and L. Nordheim, Proc. R. Soc. London A 119, 173 (1928).

[21] N. Barov, K. Bishofberger, J. B. Rosenzweig, J. P. Carneiro, P. Colestock, H. Edwards, M. J. Fitch, W. Hartung, and J. Santucci, in Proceedings of the 2001 Particle Accelerator Conference, Chicago, Illinois (Ref. [12]), p. 126. 